\title{
Effect of assertive outreach after suicide attempt in the AID (assertive intervention for deliberate self harm) trial: randomised controlled trial
}

\author{
(c) $(1)$ (8) OPEN ACCESS
}

\author{
Britt Morthorst research assistant ${ }^{1}$, Jesper Krogh research assistant ${ }^{1}$, Annette Erlangsen senior \\ researcher $^{12}$, Francisco Alberdi chief psychiatrist ${ }^{1}$, Merete Nordentoft professor ${ }^{1}$
}

${ }^{1}$ Research Unit, Mental Health Centre Copenhagen, Faculty of Health Sciences, University of Copenhagen, Bispebjerg Bakke 23, 2400 Copenhagen NV, Denmark; ${ }^{2}$ Department of Mental Health, Johns Hopkins School of Public Health, Baltimore, MD, USA

\begin{abstract}
Objective To assess whether an assertive outreach intervention after suicide attempt could reduce the frequency of subsequent suicidal acts, compared with standard treatment.

Design Randomised, parallel group, superiority trial with blinded outcome assessment.

Setting Outpatient intervention at one location at Copenhagen University Hospital, Denmark.

Participants Patients older than 12 years admitted to regional hospitals in Copenhagen with a suicide attempt within the past 14 days. We excluded patients diagnosed with schizophrenia spectrum disorders and patients living in institutions.

Intervention Case management through assertive outreach that provided crisis intervention and flexible problem solving. This approach incorporated motivational support and actively assisted patients to scheduled appointments to improve adherence with after-treatment as an add on to standard treatment.
\end{abstract}

Main outcome Repeated suicide attempt and death by suicide, recorded in medical records and death register at 1-year follow-up.

Results 243 patients were included. During 12 months of follow-up, $20 / 123(16 \%)$ patients in the intervention group had been registered in hospital records with subsequent suicide attempt, compared with 13/120 $(11 \%)$ in the control group (odds ratio $1.60,95 \%$ confidence interval 0.76 to 3.38; $P=0.22)$. By contrast, self reported data on new events showed $11 / 95(12 \%)$ in the intervention group versus $13 / 74(18 \%)$ in the control group $(0.61,0.26$ to $1.46 ; P=0.27)$. By imputing missing data on the selfreported outcomes, we estimated $15 / 123$ (12\%) events in the intervention group and $23 / 120(19 \%)$ in the control group $(0.69,0.34$ to 1.43; $P=0.32)$.

Conclusion Assertive outreach showed no significant effect on subsequent suicide attempt. The difference in rates of events between register data and self reported data could indicate detection bias.
Trial registration ClinicalTrials.gov NCT00700089.

\section{Background}

Suicidal behaviour is a serious worldwide public health problem; a million suicides are estimated to occur each year. ${ }^{12}$ Attempted suicide is under recorded. ${ }^{34}$ Nevertheless, the number of non-fatal episodes is estimated to be 10 to 40 times higher than that of fatal episodes. ${ }^{15}$ Suicide attempts occur most frequently among young women ${ }^{6}$ and an increase in the rate of suicide attempt has been noted internationally, especially among women aged 15 to 24 years. $^{7-9}$

Besides mental illness and substance abuse, previous suicide attempt is the most prominent predictor of subsequent suicidal behaviour. ${ }^{10}$ Median rates of repeated suicide attempt are $16 \%$ for non-fatal and $2 \%$ for fatal episodes within a year of the index attempt. ${ }^{11}{ }^{12}$ A European multicentre study previously recorded repetition rates of up to $30 \%$ within the first year. ${ }^{13}$ Recent studies have presented similar repetition rates for adolescents. ${ }^{14}$ The first month after a suicide attempt carries an especially high risk of repetition. ${ }^{15} 16$

Results from randomised clinical trials of treatment after suicide attempt, synthesised in systematic reviews, are heterogeneous in interventions and effect. ${ }^{6}{ }^{17} 18$ Reviews of psychosocial interventions, including a Cochrane publication and guidelines from the National Institute for Health and Clinical Excellence in England and Wales (NICE), have not found conclusive evidence on treatment strategies for this high risk group. ${ }^{17}$ 19-22 Patients with suicidal behaviour have been characterised as difficult to engage in after-treatment. ${ }^{23}$ Various trials of interventions offering help after contact with emergency rooms have focused on treatment accessibility and adherence to after-treatment, to optimise the treatment chain for both adults and adolescents. ${ }^{18} 242517$ 19-22 To reduce the gap between treatment of suicide attempt and after-care, a specialised prevention team 
in Norway provided during a 23 year period a flexible, assertive, and individually shaped treatment to people who attempted suicide without severe mental illness. ${ }^{26}$ When it compared patients who received support from the prevention team with those who did not, the study found no significant difference in risk of repeating suicide at 12 months (adjusted odds ratio 0.86 ; $95 \%$ confidence interval 0.57 to 1.30 ). The study, however, did not have a randomised design and could therefore have been influenced by selection bias. A recent randomised trial ${ }^{27}$ evaluated the Norwegian model by offering six to eight assertive and motivational consultations, while a similar control group received standard care from a general practitioner. The study, which included 133 patients, found a significant difference in favour of the intervention compared with standard treatment with rates of repeat suicide attempt being $9 \% v 22 \%$; $\mathrm{P}=0.04$ ). However, the power of the study was limited. To confirm these promising findings, our objective was to replicate the study with higher power as a randomised, parallel group, superiority trial.

The aim of this study was to investigate whether an assertive outreach intervention assigned after suicide attempt could reduce the frequency of subsequent suicidal acts, compared with standard treatment.

\section{Methods}

The assertive intervention for deliberate self harm (AID) trial was designed as a pragmatic, randomised, parallel group, superiority trial with blinded outcome assessment. The trial protocol was approved by the local ethics committee (journal number KF-01 271146), the Danish Data Protection Agency (journal number 2011-41-6398), and registered at clinicalTrials.gov (identifier NCT00700089). It was undertaken at a single location at the Copenhagen University Hospital, Copenhagen, Denmark.

\section{Participants}

Participants were patients admitted after a suicide attempt to acute emergency units, intensive care units, paediatric units, and psychiatric emergency rooms in six regional hospitals in the catchment area of Copenhagen. We made referral agreements through personal meetings with units in charge of standard treatment provided to patients with suicide attempts. We distributed pocket sized laminated sheets with inclusion and exclusion criteria to doctors in somatic and psychiatric units. Children and adolescents were referred to the AID trial from the hospital conducting the trial and from other hospitals treating children, in addition to standard procedures for psychiatric consultation. General practitioners who contacted hospitals to refer possible patients with recent suicide attempt were also informed about the trial.

\section{Inclusion criteria}

We included male and female patients aged 12 years and older with a suicide attempt within the past 14 days who were able to read and understand the informed consent statement. We made one exception with regard to the age criteria; a female patient was included shortly before her twelfth birthday.

All the suicide attempts of included patients fulfilled the WHO definition: "An act with a non-fatal outcome, in which an individual deliberately initiates a non-habitual behaviour that without intervention from others will cause self harm, or deliberately ingests a substance in excess of the prescribed or generally recognized therapeutic dosage, and which is aimed at realizing changes which the subject desired via the actual or expected physical consequences., ${ }^{28}$ Patients presenting with self injuries, such as cutting, were only included if they also met the definition of non-habitual behaviour. This was verified for each single case through patients' self reports and medical records. Patients diagnosed with severe personality disorders, including eating disorders, were invited to join the study, as were patients with alcohol misuse or with no offer of subacute treatment meeting the need for suicide prevention.

\section{Exclusion criteria}

We excluded patients admitted to a psychiatric ward for more than 14 days after the index attempt. This enabled us to investigate the effect of the intervention for high risk patients in subacute phases. We also excluded patients who had been diagnosed with schizophrenia spectrum disorders (ICD-10: F20-29), severe depression (ICD-10: F32.2, F32.3, F33.2, and F33.3.), severe bipolar disorder (ICD 10: F30 and F31), and severe dementia (ICD 10: F0-9), because intensive outreach teams are already implemented as standard care for these patients in Denmark. Furthermore, patients with severe depression or bipolar disorder and suicidal behaviour would probably need inpatient care and would not be eligible for outpatient treatment. We also excluded patients who were receiving outreach services from social service agencies or living in institutions, to prevent mixing different effects and avoid confounders. ${ }^{29}$

\section{Standard treatment}

The standard treatment consisted of referral to a range of different treatment modalities, dependent on the diagnosis and the clinical and social conditions of the patient. While the patient was still being treated for the self injury, a routine psychiatric evaluation would often be used to determine whether he or she should be referred for further treatment-for example, general practitioner, psychological therapy, or treatment for alcohol abuse. In the standard treatment there was no procedure to ensure compliance with the recommended treatment. For the most part, the patients were responsible for contacting the treatment provider to which they were referred. As a part of the standard treatment, the Centre of Excellence in Suicide Prevention Copenhagen offered six to eight therapeutic sessions to patients who did not abuse substances and were not receiving other ongoing treatments. A psychologist provided these sessions as short term therapy with risk assessment tools from the Collaborative Assessment and Management of Suicidality (CAMS) approach. ${ }^{30}{ }^{31}$ This option of after-treatment was available to patients in both groups. Pharmacological treatment of any kind was continued or prescribed in both groups when relevant.

\section{Intervention}

The AID intervention was provided as case management with crisis intervention and flexible, problem solving, assertive outreach through motivational support and actively assisting patients to and from scheduled appointments, to improve compliance with after-treatment. The intervention represented eight to 20 flexible outreach consultations performed by specialised nurses (AID managers) over 6 months in addition to standard treatment. The first consultation was offered a few days after discharge and focused on acute prevention strategies, such as developing a crisis intervention plan in case of relapse. Subsequent consultations established a professional relationship, addressed problem solving strategies, and undertook specific actions. To ensure continuity, the same AID manager contacted the patient throughout the 6 months of the intervention. 
Motivation towards after-treatment was stressed as a core feature and referral was organised. Consultations were provided equally as home visits or meetings in cafes, as preferred, as well as meetings with healthcare, official, and social services to accommodate individual needs. The AID manager was responsible for maintaining contact, but the patient was asked to make contact between consultations if necessary. Telephone calls and text messages were frequently used to strengthen the alliance. The manager offered mediating family consultations to adolescents and their relatives, and suggested the involvement of social or professional networks irrespective of age. During relapse or stressful life events, frequency of contact was intensified, including availability of contact out usual hours to prevent subsequent suicidal behaviour. If the patient had severe suicidal impulses, the manager asked him or her to take a prepaid taxi to the psychiatric emergency room if all other items on the crisis intervention plan had failed in changing the impulses or providing relief of psychic pain. A minimum of four personal contacts was defined as adherence to the AID intervention; additional phone calls and text messages were not defined. Cafe visits did not count as crisis interventions. If, however, the patient revealed severe suicidal impulses during a cafe visit, the manager would usually prolong contact to cover decisions of crisis management.

\section{Staff requirements}

The intervention staff (AID managers) were highly qualified psychiatric nurses with specialised training in suicidology. Regular consultations were done with the supervising chief psychiatrist and research assistant to ensure adherence to the research protocol. The target for maximum caseload for each AID manager was 20 patients in active intervention treatment at any time. If a patient died by suicide, the AID manager assigned the patient would immediately be relieved from duty for a period of time.

\section{Outcome}

We interviewed patients at baseline to obtain information on socio-demographic variables and method used for the index suicide attempt. The outcomes of interest were repeated suicide attempt and death by suicide with a follow-up period of one year after inclusion date. We obtained information on these events through ICD-10 diagnoses (recorded as part of standardised hospital registration procedure) and review of medical records. Additionally, we collected self reported data on events within the first year of follow-up, through telephone interviews with patients. Patients who could not be reached by phone after persistent attempts were contacted by regular mail.

\section{Blinding}

An external medical evaluation committee conducted a blinded outcome assessment using medical records. Owing to the nature of the study design, the intervention staff were not blinded. The researcher conducting the analyses on self reported outcomes was also not blinded.

\section{Statistical power}

On the basis of previous studies, ${ }^{27}$ we estimated an expected repetition risk of $30 \%$ in the standard group and $15 \%$ in the intervention group within one year of the index attempt. With an $\alpha$ value of 0.05 , we required 120 patients randomised to each treatment arm to reach a power of $80 \% .^{32}$

\section{Randomisation}

Computer randomisation was done by an independent research assistant at the Mental Health Centre Copenhagen, stratified by whether the patient had previously attempted suicide (first attempt $v$ previous attempt), previous psychiatric contacts or hospitalisation (none $v$ previous contacts), and alcohol consumption at time of suicide attempt (none $v$ alcohol consumption). The AID managers were informed of the allocation by phone and the patients were immediately informed of the outcome. The randomisation procedure ensured adequate sequence generation and allocation concealment.

\section{Statistics and data analysis}

We assessed frequencies, means, and standard deviations of socio-demographic and patient characteristics and tested differences between groups with $\chi^{2}$ or independent sample $t$ tests. We calculated the log odds of a patient having a recorded or self reported new suicide attempt in the 12 months following the index attempt. In subsequent planned analyses, we adjusted for possible baseline differences relevant for the primary outcome. All patients were included in the analysis regardless of subsequent adherence to treatment, according to the intention to treat principle. All statistical tests were two sided and $\mathrm{P}$ values less than 0.05 were considered statistically significant. We imputed missing values for the self reported outcome as a dependent variable using multiple imputations by applying a linear regression model with the following predictor variables: allocation, suicide attempt prior to index attempt, alcohol intoxication at index attempt, psychiatric contact prior to index attempt, unemployment, antidepressant medication, and discharge from a psychiatric hospital within the previous four weeks. The imputation was conducted using 100 imputations and 20 iterations. The pooled estimates from these imputations were subsequently used for analysis. The statistical software package SPSS version 19.0 was used for the analyses.

\section{Ethical considerations}

We obtained written consent from all participants after providing them with both oral and written information about the conditions of participating in scientific research in general and with specific details of the AID trial. Age specific consent forms were developed for children and adolescents. Written consent from both custody holders was required and obtained for children aged 11-14 years. Adolescents aged 15-17 years were allowed to decide and give written consent themselves as long as their parents were informed, according to the Danish Committee on Biomedical Research Ethics.

\section{Results \\ Patients}

Between November 2007 and March 2010, 305 patients were invited to participate in the trial (figure $1 \Downarrow$ ). During the 29 months, 243 patients (184 women; 76\%) were included. The mean age was 31 years in the control group (standard deviation (SD) 12) and 31 years in the intervention group (SD 14; range 11-69 years). Of those included, 67 (28\%) were aged 20 years or younger. The only differences between participants at baseline were that patients in the intervention group were more frequently being treated with antidepressants at inclusion $\left(\chi^{2} 6.76\right.$, df 1 , $\mathrm{P}=0.03)$ and more frequently used narcotics as method of suicide attempt, compared with controls $(\mathrm{P}=0.05)$ (table $1 \Downarrow)$. Self reported data on subsequent events were obtained from 169/243 patients $(70 \%)$. Significantly more patients provided this 
information in the intervention group $(95 / 123,77 \%)$ than in the control group $\left(74 / 120,62 \%, \chi^{2} 7\right.$, df $\left.1 ; \mathrm{P}=0.01\right)$.

\section{Service use in the intervention group}

The service offered in the AID-intervention was flexible, with a median of 9 (interquartile range (IQR) 6-12) home consultations including cafe visits, $1(0-3)$ attendances to healthcare services, 12 (7-21) phone calls to patients and relatives, and 2.5 (0-5) and 5 ( 0 to 8$)$ phone calls to healthcare and social services, respectively. The median number of text messages sent was 17 (IQR 8 to 30). In total, 91 crisis interventions were provided. These interventions included phone calls when severe suicidal ideation or impulses were present and emergency calls when either somatic or psychiatric admission was requested. Five patients did not receive the allocated intervention because they withdrew from the study.

\section{Primary outcome}

During the 12 months' follow-up, we found that 20/123 (16\%) patients in the intervention group had been registered in hospital records with subsequent attempts, compared with 13/120 (11\%) in the control group (odds ratio (OR) 1.60, 95\% confidence interval 0.76 to $3.38 ; \mathrm{P}=0.22$ (table $2 \Downarrow$ ). Adjusting the primary analysis with the use of antidepressants at baseline did not markedly alter the results $(1.67,0.78$ to $3.58 ; \mathrm{P}=0.19)$. Our analysis suggested no association between antidepressants and allocation $(\mathrm{P}=0.48)$.

By contrast, self reported data on new events showed 11/95 $(12 \%)$ in the intervention group versus $13 / 74$ (18\%) in the control group (OR $0.61,95 \%$ confidence interval 0.26 to 1.46 ; $\mathrm{P}=0.27$; table 2). Seven patients (four in the intervention group versus three in standard care) were identified with a subsequent suicide attempt in the hospital records although the patients themselves had reported none. Correspondingly, nine patients (one in the intervention group versus eight in standard care) themselves reported subsequent attempts that were not listed in the hospital records. Analysis of missing self reported data based on baseline information about relevant risk factors (sex, age, unemployment, suicide attempts prior to index attempt, prior psychiatric admission, and alcohol consumption at index attempt) assumed data were missing at random. By imputing missing data on the self reported outcome as described above we estimated $15 / 123(12 \%)$ in the intervention group and 23/120 (19\%) in the control group $(\mathrm{OR}=0.69,95 \%$ confidence interval 0.34 to $1.43 ; \mathrm{P}=0.32$; table 2 ).

We identified two deaths due to causes other than suicide; one in the intervention group and one in the control group. One patient died by suicide in the intervention group within two weeks of the index attempt.

Combining the self reported outcome with the hospital records in post-hoc analyses did not markedly alter results $(\mathrm{OR}=0.97$; 95\% confidence interval 0.50 to $1.89 ; \mathrm{P}=0.93$ ).

Additional analyses using attendance to healthcare services as the outcome did not show more health care contacts by participants in the intervention compared with the standard group $(\mathrm{OR}=0.5795 \%$ confidence interval 0.29 to $1.14 ; \mathrm{P}=0.11)$. Similarly, attendance to social services was similar between in the two groups $(1.01,0.61$ to $1.68 ; \mathrm{P}=0.96)$.

\section{Subgroup analysis}

A separate subgroup analysis conducted by age group showed no impact of age on estimated effects of the intervention (table 2). Subgroup analyses by sex and by diagnoses of mild to moderate depression and personality disorders did not obtain differences in effects.

\section{Discussion}

\section{Principal findings}

This pragmatic, randomised controlled clinical trial investigated the effect of assertive outreach as a preventive treatment strategy for patients with a recent suicide attempt. The trial did not find any significant difference in repetition rates between the group receiving the intervention and the group receiving standard treatment. We noted a difference between register data and self reported data for subsequent suicide attempts, especially in the control group. The AID trial has contributed to international research by investigating an assertive approach demanded by experts and clinicians.

\section{Strengths and weaknesses of the trial}

A strength of the trial was the randomised design and high methodological quality, associated with a low risk of bias. ${ }^{29}$ The robustness was increased by a relatively large sample size and use of detailed register data to ensure that no patients were lost to follow-up. The frequency of assertive consultations and service use suggest that patients accepted the experimental intervention by adhering to the approach. The study covered a heterogeneous population, including patients with alcohol and substance abuse problems, and those diagnosed with borderline personality disorder and various other diagnoses. Both these groups are at high risk, ${ }^{12}{ }^{33}$ often with limited or already rejected treatment opportunities. The trial population was therefore similar to that seen in clinical reality, leading to high external validity of the study. Since patients diagnosed with schizophrenia spectrum disorders were excluded, the findings do not apply to these patients, but they do apply to other groups of high risk patients without psychosis.

The lack of a manual based approach for the AID intervention and the presence of various kinds of after-treatment modalities in both study groups makes replication less feasible and could affect the internal validity. Marked distinctions between the standard and intervention approaches were the assertiveness, the accessibility, and the active assistance of patients to scheduled appointments in the intervention group; these parts were all thought to be substantially relevant for this patient group. ${ }^{18-25}$ The standard treatment offered by the Centre of Excellence in Suicide Prevention Copenhagen improved during the study period and is judged to be after-treatment of good quality. As the AID intervention was an add-on, the standard treatment was also offered to patients in the intervention group, thus avoiding risk of bias. Although the study groups were located in different settings, contamination of standard treatment cannot be excluded. Different motivational factors might explain the lack of a difference between the groups in attendance of health and social services. During the study period, the Danish government's requirements for receiving sick leave benefits included mandatory frequent contact with social services; if citizens did not attend scheduled appointments, they risked losing their sick leave benefits. Also, social services often require medical documentation in order to issue sick leave benefits. Figures of attendance to health and social services in the control group do not show whether patients were adherent to service requirements.

The significantly higher proportion of patients prescribed antidepressants at baseline in the intervention group could constitute a source of bias. These patients might represent a group with more severe mental illness who might also be more 
difficult to prevent from subsequent suicidal behaviour. ${ }^{18}$ On the other hand, patients already receiving drug treatment might, arguably, be less depressed than those not receiving pharmacotherapy. ${ }^{18}{ }^{20}$ However, the analysis adjusting for this potential confounder found no indication of bias.

The relatively wide age range of the study population, which included teenagers and older people as well as people of both sexes, could be considered a potential limitation owing to the varied needs and help seeking behaviours of different groups. However, our subgroup analyses showed no effect of age or sex on the findings. The recorded rate of subsequent suicide attempts among males was slightly higher in the intervention group than in the standard group, which might give rise to speculation that males in the standard group were less inclined to seek help, leading to detection bias. However, the difference in rates between the groups was not significant.

We expected that adherence to the AID intervention would reduce subsequent suicide attempts. However, we also expected that the intervention would encourage participants to seek contact more frequently, which could have increased the likelihood that subsequent attempts were reported. The higher repetition rate shown in the hospital records might suggest that patients in the intervention arm, after subsequent attempts, called the AID manager, who then provided emergency arrangements. The weakening of differences in effect when self reported information was added to the analysis adds to this argument. ${ }^{10}$

The power calculation, in which we estimated a repetition risk of $30 \%$ in the standard group and $15 \%$ in the intervention group, could be judged to be too optimistic. The calculation relied on rates recorded in past European studies ${ }^{13}{ }^{34}$ but might not have been powered to detect smaller differences such as those present in this trial.

There was disagreement between the two data sources over the number of suicide attempts. The clinical decision to classify a self inflicted episode as a suicide attempt corresponding to a diagnosis in hospital records is often not consistent. Also, in the acute phase of treating the trauma, diagnoses from emergency departments do not always reflect the suicidal dimension of the event, ${ }^{3}$ unless the risk of suicide is ongoing. The WHO definition of suicide attempt used in this trial also leaves room for interpretation; although a blinded external evaluation committee examined doubtful cases in detail, a small number of false positives was unavoidable, especially since the medical records in some cases provided limited information.

The dissonance in number of suicide attempts between the two data sources could also have been due to differences between the groups in the participants' perception of their own need or attitudinal barriers-for example, the wish to handle problems alone, which is related to lower tendency for help seeking behaviours. ${ }^{35-37}$ Furthermore, some patients might not be completely truthful about subsequent suicide attempts in order to perform well when interviewed by the researcher, hence a risk of performance bias. On the other hand, patients who reported subsequent attempts but had no hospital record of such an event might have preferred to avoid hospital contact, because they feared being registered and potentially stigmatised later on. These factors reflect a risk in this trial of detection bias related to both underestimation and overestimation.

The multiple imputations indicated a potential underestimation based on self reported data. ${ }^{29}$ However, given that suicidal patients tend to display a lack of treatment seeking behaviour, self reported data might actually have a higher validity than register data.
Restrictions by the Danish Data Protection Agency did not allow analysis of information on patients who refused to participate in the trial. Because the inclusion criteria were pragmatic, we did not expect this to affect the external validity.

Previous studies have recommended a structured risk assessment as an important tool in suicide prevention. ${ }^{22}$ Although suicidal behaviour was routinely addressed as a pivotal point of all consultations in the AID intervention, no formal risk assessment tool was used besides the development of crisis intervention plans and individual psychoeducation.

\section{Comparison with other studies}

The result of the present trial are similar to those presented in reviews of psychosocial interventions in general. ${ }^{18-20}$ However, a study of a similar intervention by Hvid and colleagues did find an effect. ${ }^{27}$ The differences in baseline characteristics between that trial and the AID trial suggest that our study included more patients with previous suicide attempts and former psychiatric contacts. The study populations might have differed, therefore, in severity of mental illness. However, Hvid and colleagues did not provide information on diagnosis, and data on service use were only provided for the intervention group. Interestingly, both trials used same inclusion and exclusion criteria and were conducted within a few years and a radius of $5 \mathrm{~km}$. The most likely explanation for the difference in effects seems to be type one error, or that the standard treatment offered in the previous study was less intensive. Standard treatment in the previous study mainly consisted of standard care in general practice, whereas the current study was able to offer an enhanced treatment through the Centre of Excellence during the AID trial.

Baseline characteristics of the current sample resembled populations in international studies; for instance, three quarters of those who attempted suicide were women, ${ }^{6}$ one quarter were of younger age ( $<20$ years), ${ }^{8}{ }^{38}$ and overdose was the most frequent method. ${ }^{6}{ }^{16}$ Based on post-hoc analysis, the combined repetition rates based on medical records and self reported data were estimated to be $17 \%$ for the intervention group and $18 \%$ in the standard, respectively, which corresponded to repetition rates previously outlined in international reviews. ${ }^{10-12}$

\section{Possible explanations and implications for clinicians and policymakers}

Although experts, clinicians, and patients have called for an assertive approach to suicide prevention, we did not find evidence to recommend such a strategy. However, the complexity of suicidal behaviour might not be addressed adequately by accompanying patients to after-treatment if waiting lists are extensive, or if the treatment modalities are not evidence-based. By comparison with other settings, the standard treatment in our trial was of good quality, and this could affect the external validity. However, the repetition rate among recipients of standard treatment was still very high. We do not believe, therefore, that the threshold for improvements has been reached. It would have been appropriate and interesting to rate patients on relevant suicidal scales (for example, for depressive symptoms, substance abuse, and self efficacy) at baseline and follow-up, to detect any difference in secondary outcomes in relation to treatment modalities.

Extensive information is available on risk factors for suicidal behaviour, ${ }^{6} 1039$ but less is known about recommendations for psychosocial treatment. ${ }^{19}{ }^{20}$ Evidence of benefit has been shown for cognitive behaviour therapy ${ }^{2122}$ and for psychosocial treatment within a family context for adolescents. ${ }^{40}$ However, 
this evidence relates to specific subgroups and has yet to be replicated in heterogeneous populations.

A remarkable relative difference was noted between the number of registered and self reported repeated suicide attempts among patients in the standard treatment. Suicide attempts are probably under recorded in general. ${ }^{156}$ However, it is worrisome that these people did not seek help despite being enrolled in an ongoing treatment programme. The estimate of multiple imputations on self reported data suggested that there could be even more patients with subsequent unrecorded events in both groups. The long term consequences of untreated deliberate self harm are unknown, but are certainly a potential risk factor.

Patients attempting suicide constitute a heterogeneous group, differing in age, life conditions, and risk factors. Prevention strategies targeting repeated suicide attempts need to address most of the raised risk factors by providing crisis intervention, being assertive and accessible, and at the same time offering individually shaped support. The AID intervention aimed to incorporate all these essential elements and yet failed to reduce the repetition frequency in a large, representative sample. Future research could target subgroups with even more specialised therapeutic interventions, such as specialised cognitive behavioural therapy, or could focus on primary prevention strategies addressing risk and vulnerability before the first suicide attempt.

We thank all included patients and relatives, intervention staff Agnete Birktoft and Hanne Frandsen, all collaborating partners in intensive units both somatic and psychiatric, Centre of Excellency in Suicide Prevention, social services, external psychiatrists, and psychologists. We thank the investigators Hvid and Wang for their collaboration.

Contributors: $\mathrm{MN}$ was primary investigator. $\mathrm{BM}$ and $\mathrm{MN}$ developed the research protocol, designed the study, acquired funding, carried out the intervention, and gathered data. FA was administrative collaborator and consulting physician for the research assistant. FA, AE, and MN were the blinded external evaluation committee. BM and JK constructed the database and conducted the statistical analysis. All authors contributed to writing and revising the manuscript. BM and $\mathrm{MN}$ are guarantors.

Funding: The study received funding from the Ministry of Health and Internal Affairs, Denmark, the National Board of Social Services, an independent subdivision of The Ministry of Social Affairs and Integration, TrygFonden, and Aase og Ejnar Danielsens Foundation.

Competing interests: All authors have completed the ICMJE uniform disclosure form at www.icmje.org/coi_disclosure.pdf (available on request from the corresponding author) and declare: the study received funding from the Ministry of Health and Internal Affairs, Denmark, the National Board of Social Services, an independent subdivision of The Ministry of Social Affairs and Integration, TrygFonden, and Aase og Ejnar Danielsens Foundation; no financial relationships with any organisations that might have an interest in the submitted work in the previous three years; no other relationships or activities that could appear to have influenced the submitted work.

Ethical approval: The trial was approved by the Danish Ethic Committee (journal number KF-01 271146) and by the Danish Data Protection Agency (journal number 2011-41-6398). All participating patients gave written informed consent.

Data sharing: No additional data available.

WHO. Suicide rates per 100000 by country, year and sex. 2009. www.who.int/mental health/prevention/suicide_rates/en.

2 Krug EG, Mercy JA, Dahlberg LL, Zwi AB. The world report on violence and health. Lancet 2002;360:1083-8.

3 Nordentoft M, Sogaard M. Registration, psychiatric evaluation and adherence to psychiatric treatment after suicide attempt. Nord J Psychiatry 2005;59:213-6.

4 Bertolote JM, Fleischmann A. Suicidal behavior prevention: WHO perspectives on research. Am J Med Genet C Semin Med Genet 2005;133C:8-12.
5 Bertolote JM, Fleischmann A. A global perspective on the magnitude of suicide mortality. The Oxford textbook of suicidology and suicide prevention: a global perspective . Oxford: 2009: 91-8.

6 Hawton K, van Heeringen K. Suicide. Lancet 2009;373:1372-81.

7 Qin P, Mortensen PB, Agerbo E, Westergaard-Nielsen N, Eriksson T. Gender differences in risk factors for suicide in Denmark. Br J Psychiatry 2000;177:546-50.

8 Hawton K, Arensman E, Wasserman D, Hulten A, Bille-Brahe U, Bjerke T, et al. Relation between attempted suicide and suicide rates among young people in Europe. J Epidemiol Community Health 1998;52:191-4.

9 Bridge JA, Goldstein TR, Brent DA. Adolescent suicide and suicidal behavior. J Child Psychol Psychiatry 2006;47:372-94

10 Borges G, Nock MK, Haro Abad JM, Hwang I, Sampson NA, Alonso J, et al. Twelve-month prevalence of and risk factors for suicide attempts in the World Health Organization World Mental Health Surveys. J Clin Psychiatry 2010;71:1617-28.

11 Owens D, Horrocks J, House A. Fatal and non-fatal repetition of self-harm. Systematic review. Br J Psychiatry 2002;181:193-9.

12 Nordentoft M. Prevention of suicide and attempted suicide in Denmark. Epidemiological studies of suicide and intervention studies in selected risk groups. Faculty of Health Sciences, Copenhagen University Hospital, Psychiatric Center Bispebjerg, 2007.

13 Schmidtke A, Bille-Brahe U, DeLeo D, Kerkhof A, Bjerke T, Crepet P, et al. Attempted suicide in Europe: rates, trends and sociodemographic characteristics of suicide attempters during the period 1989-1992. Results of the WHO/EURO Multicentre Study on Parasuicide. Acta Psychiatr Scand 1996;93:327-38.

14 Brent DA, Greenhill LL, Compton S, Emslie G, Wells K, Walkup JT, et al. The treatment of adolescent suicide attempters study (TASA): predictors of suicidal events in an open treatment trial. J Am Acad Child Adolesc Psychiatry 2009;48:987-96.

15 Cedereke M, Ojehagen A. Prediction of repeated parasuicide after 1-12 months. Eur Psychiatry 2005;20:101-9.

16 Qin P, Jepsen P, Norgard B, Agerbo E, Mortensen PB, Vilstrup H, et al. Hospital admission for non-fatal poisoning with weak analgesics and risk for subsequent suicide: a population study. Psychol Med 2009;39:1867-73.

17 Hawton K, Arensman E, Townsend E, Bremner S, Feldman E, Goldney R, et al. Deliberate self harm: systematic review of efficacy of psychosocial and pharmacological treatments in preventing repetition. BMJ 1998;317:441-7.

18 Mann JJ, Apter A, Bertolote J, Beautrais A, Currier D, Haas A, et al. Suicide prevention strategies: a systematic review. JAMA 2005;294:2064-74.

19 Crawford MJ, Thomas O, Khan N, Kulinskaya E. Psychosocial interventions following self-harm: systematic review of their efficacy in preventing suicide. Br J Psychiatry 2007;190:11-7.

20 Hawton K, Townsend E, Arensman E, Gunnell D, Hazell P, House A, et al. Psychosocial versus pharmacological treatments for deliberate self harm. Cochrane Database Syst Rev 2000;2:CD001764.

21 NICE. Self-harm: the short-term physical and psychological management of secondary prevention of self-harm in primary and secondary care. Clinical guideline CG16. National Institute for Health and Clinical Excellence. 2004. www nice.org.uk/CG16.

$22 \operatorname{Tim~K,~Clare~T,~Henna~B,~Melissa~C,~Navneet~K.~Longer~term~management~of~self~harm:~}$ summary of NICE guidance. BMJ 2011;343.

23 Lizardi D, Stanley B. Treatment engagement: a neglected aspect in the psychiatric care of suicidal patients. Psychiatr Serv 2010;61:1183-91.

24 Rotheram-Borus MJ, Piacentini J, Van RR, Graae F, Cantwell C, Castro-Blanco D, et al. Enhancing treatment adherence with a specialized emergency room program for adolescent suicide attempters. J Am Acad Child Adolesc Psychiatry 1996;35:654-63.

25 Spirito A, Boergers J, Donaldson D, Bishop D, Lewander W. An intervention trial to improve adherence to community treatment by adolescents after a suicide attempt. J Am Acad Child Adolesc Psychiatry 2002;41:435-42.

26 Johannessen HA, Dieserud G, De LD, Claussen B, Zahl PH. Chain of care for patients who have attempted suicide: a follow-up study from Baerum, Norway. BMC Public Health 2011;11:81.

27 Hvid M, Vangborg K, Sorensen HJ, Nielsen IK, Stenborg JM, Wang AG. Preventing repetition of attempted suicide-II. The Amager Project, a randomized controlled trial. Nord J Psychiatry 2011;65:292-8.

28 Platt S, Bille-Brahe U, Kerkhof A, Schmidtke A, Bjerke T, Crepet P, et al. Parasuicide in Europe: the WHO/EURO multicentre study on parasuicide. I. Introduction and preliminary analysis for 1989. Acta Psychiatr Scand 1992;85:97-104.

29 Gluud LL. Bias in clinical intervention research. Am J Epidemiol 2006;163:493-501.

30 Ellis TE, Allen JG, Woodson H, Frueh BC, Jobes DA. Implementing an evidence-based approach to working with suicidal inpatients. Bull Menninger Clin 2009;73:339-54.

31 Jobes DA, Wong SA, Conrad AK, Drozd JF, Neal-Walden T. The collaborative assessment and management of suicidality versus treatment as usual: a retrospective study with suicidal outpatients. Suicide Life Threat Behav 2005;35:483-97.

32 Dupont WD, Plummer WD, Jr. Power and sample size calculations. A review and computer program. Control Clin Trials 1990;11:116-28.

33 Mortensen PB, Agerbo E, Erikson T, Qin P, Westergaard-Nielsen N. Psychiatric illness and risk factors for suicide in Denmark. Lancet 2000;355:9-12.

34 Hvid M, Wang AG. Preventing repetition of attempted suicide I. Feasibility (acceptability, adherence, and effectiveness) of a Baerum-model like aftercare. Nord J Psychiatry 2009;63:148-53

35 Pagura J, Fotti S, Katz LY, Sareen J. Help seeking and perceived need for mental health care among individuals in Canada with suicidal behaviors. Psychiatr Serv 2009;60:943-9.

36 Bruffaerts R, Demyttenaere K, Hwang I, Chiu WT, Sampson N, Kessler RC, et al. Treatment of suicidal people around the world. Br J Psych 2011;199:64-70.

37 Gulliver A, Griffiths K, Christensen H. Perceived barriers and facilitators to mental health help-seeking in young people: a systematic review. BMC Psychiatry 2010;10:113.

38 Pelkonen M, Marttunen M. Child and adolescent suicide: epidemiology, risk factors, and approaches to prevention. Paediatr Drugs 2003;5:243-65.

39 Bergen H, Hawton K, Waters K, Cooper J, Kapur N. Epidemiology and trends in non-fatal self-harm in three centres in England, 2000 to 2007. Br J Psychiatry 2010:197:493-8.

40 Daniel SS, Goldston DB. Interventions for suicidal youth: a review of the literature and developmental considerations. Suicide Life Threat Behav 2009;39:252-68.

Accepted: 11 July 2012 


\section{What is already known on this topic}

- Suicide attempt is often associated with subsequent suicidal behaviour, but patients can be difficult to engage in after-treatment

- A previous study with limited power suggested that assertive and motivational consultations could reduce the rate of repeated suicide attempts

\section{What this study adds}

- This randomised clinical trial did not find evidence to recommend an assertive intervention over standard care for patients after suicide attempt

- Patients allocated to the intervention were more likely than controls to be hospitalised and treated for subsequent suicide attempts

- Patients were compliant with the intervention

This is an open-access article distributed under the terms of the Creative Commons Attribution Non-commercial License, which permits use, distribution, and reproduction in any medium, provided the original work is properly cited, the use is non commercial and is otherwise in compliance with the license. See: http://creativecommons.org/licenses/bync/2.0/ and http://creativecommons.org/licenses/by-nc/2.0/legalcode. 


\section{Tables}

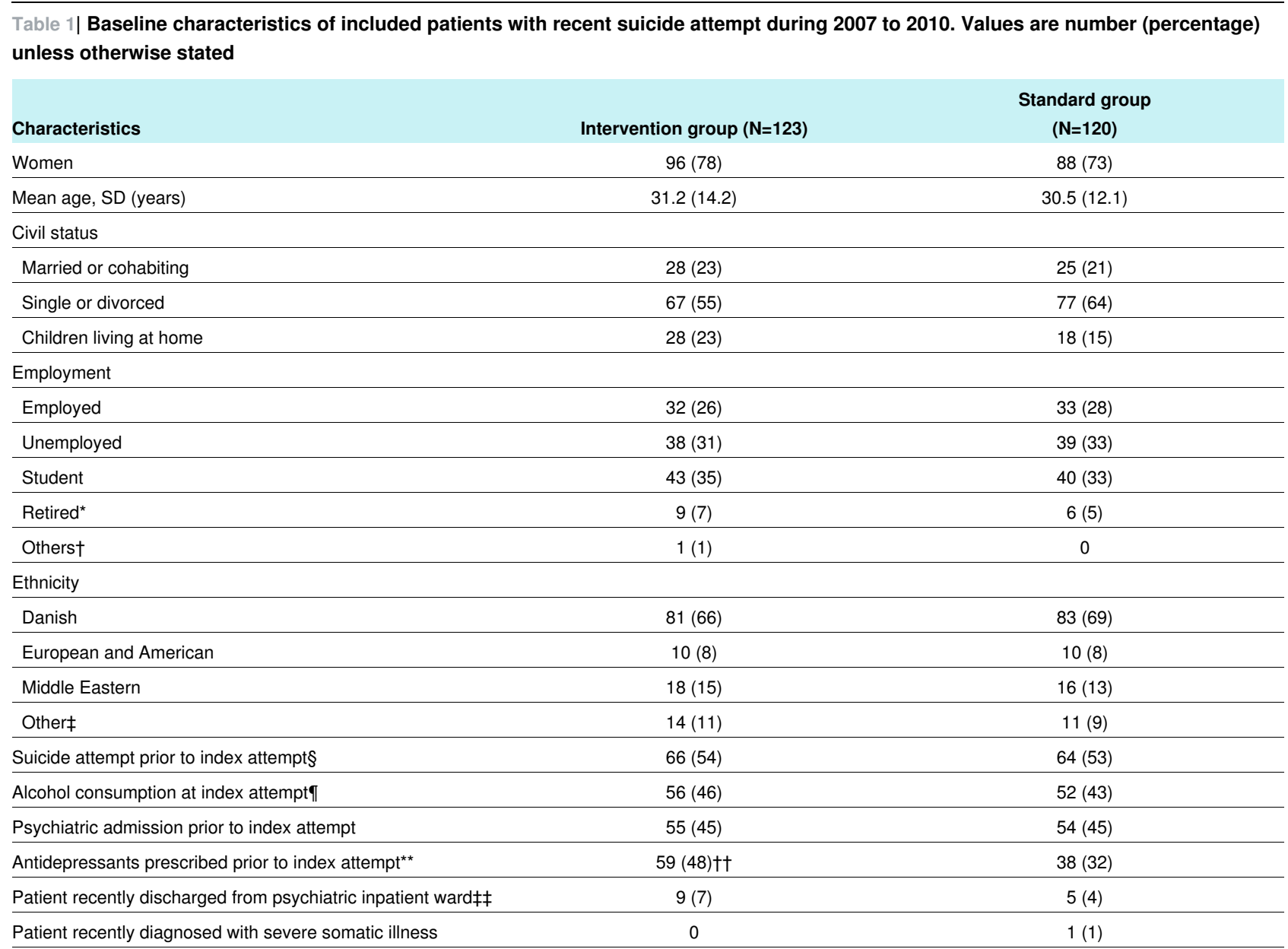

${ }^{*}$ Patients receiving pensions due to early retirement, disability, or retirement.

tIncluding housewives and other non-employed people.

łIncluding eastern European, Asian, African, and people from the Arctic.

§According to WHO definitions.

INot necessary diagnosed with substance abuse, but alcohol intake in relation to index attempt.

${ }^{*}$ Data missing for two patients (one standard, one intervention)

†† $\mathrm{P}<0.05$.

$\ddagger \ddagger$ Patients discharged from psychiatric inpatient ward within four weeks preceding index attempt. Data missing for five patients (three standard, two intervention). 
Table 2| Number of patients with subsequent suicide attempt at one year follow-up, by source of data on new events and by subgroup. Values are number of patients with one or more new events/number in group with available data (percentage) unless otherwise stated

\begin{tabular}{|c|c|c|c|c|}
\hline & Intervention group & Standard group & $P$ value & Odds ratio $(95 \% \mathrm{Cl})$ \\
\hline \multicolumn{5}{|l|}{ Data source } \\
\hline Hospital recorded & $20 / 123(16)$ & $13 / 120(11)$ & 0.22 & $1.60(0.76$ to 3.38$)$ \\
\hline Self reported & $11 / 95(12)$ & $13 / 74(18)$ & 0.27 & $0.61(0.26$ to 1.46$)$ \\
\hline Estimated by multiple imputations & $15 / 123(12)$ & 23/120 (19) & 0.32 & 0.69 (0.34 to 1.43$)$ \\
\hline \multicolumn{5}{|l|}{ Subgroup analysis } \\
\hline \multicolumn{5}{|l|}{ Age } \\
\hline $11-19$ & $7 / 30(23)$ & $7 / 26(27)$ & 0.76 & 0.83 (0.25 to 2.77$)$ \\
\hline 20-39 & $10 / 65(15)$ & $4 / 66(6)$ & $0.10^{*}$ & $2.82(0.84$ to 9.50$)$ \\
\hline $40+$ & $3 / 28(11)$ & $2 / 28(7)$ & $1.00^{*}$ & $1.56(0.24$ to 10.14$)$ \\
\hline \multicolumn{5}{|l|}{ Sex } \\
\hline Male & $7 / 27(26)$ & $2 / 32(6)$ & $0.07^{*}$ & 5.25 (0.99 to 27.90$)$ \\
\hline Female & $13 / 96(14)$ & $11 / 88(13)$ & 0.83 & 1.10 (0.46 to 2.59$)$ \\
\hline \multicolumn{5}{|l|}{ Diagnosis } \\
\hline Mild to moderate depression $†$ & $19 / 110(17)$ & $13 / 112(12)$ & 0.23 & $1.59(0.74$ to 3.40$)$ \\
\hline Personality disorder $\ddagger$ & $9 / 110(8)$ & $7 / 112(6)$ & 0.58 & 1.34 (0.48 to 3.73$)$ \\
\hline
\end{tabular}

*Fisher's exact test.

†Diagnosed at time of inclusion (ICD-10: F32.0-1, F32.8-9, F33.0-1, F33.4-9, F34-39).

‡Diagnosed at time of inclusion (ICD-10: F60-69). 


\section{Figure}

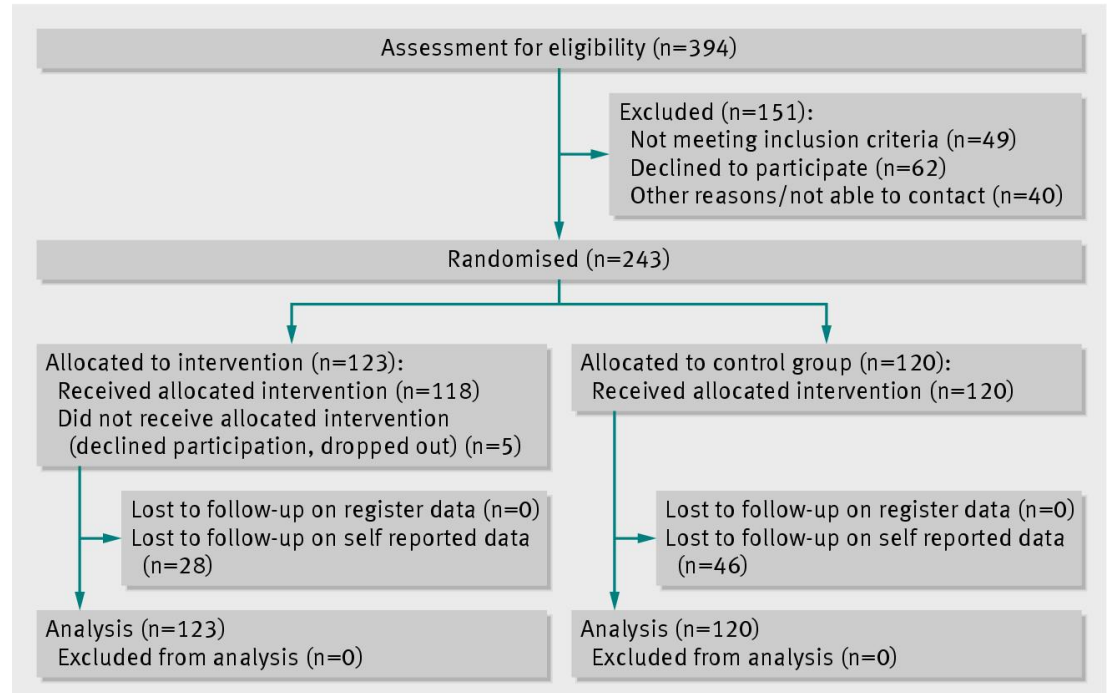

Fig 1 The AID trial profile 